\title{
Intracolonic infusion of faecal supernatant fraction from patients with diarrhoea-predominant irritable bowel syndrome evokes visceral hyperalgaesia in mice: reversal by a probiotic treatment
}

\author{
A. Ait-Belgnaoui ${ }^{1}$, H. Eutamene ${ }^{1}$, L. Ferrier ${ }^{1}$, H. Durand $^{2}$, J. Fioramonti $^{1}$, L. Bueno $^{1}$ \\ and V. Theodorou-Bayle ${ }^{1}$ \\ ${ }^{1}$ UMR 1054 INRA/EI-Purpan, Toulouse, France and ${ }^{2}$ LALLEMAND, Toulouse, France
}

Irritable bowel syndrome (IBS), a functional gastrointestinal disorder affecting 5-20\% of the population worldwide, is characterized by abdominal pain, altered bowel habits, 'low-grade' mucosal inflammation, impaired intestinal permeability and quantitative alterations in commensal microbiota. Faecal supernatant fractions from patients with diarrhoea-predominant IBS (IBS-D) have three to four times higher protease activity, and mucosal application of these supernatant fractions in mice induces visceral hyperalgaesia that is prevented by serine protease inhibitors ${ }^{(1)}$. Despite an improvement in IBS symptoms by some probiotics, their mechanism of action remains poorly understood. Consequently, the aim was to evaluate in mice whether (a) a probiotic (Lactobacillus farciminis; Lf) prevents visceral hypersensitivity induced by intracolonic infusion of an IBS-D faecal supernatant fraction and (b) this effect is linked to the ability of $L f$ to reduce serine protease activity.

Four groups of ten mice received orally for $15 \mathrm{~d}$ either $10^{9} \mathrm{CFU} L f / \mathrm{d}$ (groups 1 and 2) or saline ( $\left.9 \mathrm{~g} \mathrm{NaCl} / \mathrm{l}\right)$; groups 3 and 4$)$. Mice were equipped with $\mathrm{NiCr}$ electrodes in the abdominal muscle, and electromyographic (EMG) activity was recorded as an index of visceral pain. Noxious colo-rectal distensions (CRD) were performed using an embolectomy probe inserted into the rectum and inflated from 0 ml to $0.12 \mathrm{ml}$, in $0.02 \mathrm{ml}$ steps, each lasting $10 \mathrm{~s}$. A faecal supernatant fraction from IBS-D faecal materials (groups 2 and 4 ) or vehicle (groups 1 and 3) was infused intracolonically $(300 \mu \mathrm{l})$, and CRD was performed $1 \mathrm{~h}$ after the infusion. Serine protease activity of an IBS-D faecal supernatant fraction was assessed in vitro in the presence of $L f$ growth and $L f$-free culture medium.

Intracolonic administration of IBS-D faecal supernatant fractions significantly increased the abdominal EMG response at $0.08 \mathrm{ml}$ and $0.1 \mathrm{ml} \mathrm{CRD}$ volumes when compared with the control. $L f$ treatment prevented the hypersensitivity induced by infusion of an IBS-D faecal supernatant fraction (amplitude of contraction (mV/s) at $0.08 \mathrm{ml} \mathrm{CRD} \mathrm{volume;} 139$ (SE 6) v. 94 (SE 10); $P<0.05$ ). Serine protease activity of an IBS-D faecal supernatant fraction was inhibited by $L f$ fresh growth medium (LGM) or filtered LGM to obtain bacteria-free culture medium $(-69 \%$ and $-71 \%$ respectively; $P<0.01)$.

$L f$ or its metabolites reduce the serine protease activity of IBS-D faecal supernatant fractions, and $L f$ treatment prevents proteasemediated visceral hyperalgaesia induced by IBS-D faecal supernatant fraction. These results suggest the involvement of a protease inhibitory factor released by this strain in the beneficial effect observed.

1. Gecse C, Roka R, Ferrier L et al. (2008) Gut (In the Press). 